\title{
Hepatic and glucagon-like peptide-1- mediated reversal of diabetes by glucagon receptor antisense oligonucleotide inhibitors
}

Kyle W. Sloop, ${ }^{1}$ Julia Xiao-Chun Cao, ${ }^{1}$ Angela M. Siesky, ${ }^{1}$ Hong Yan Zhang, ${ }^{1}$ Diane M. Bodenmiller, Amy L. Cox, ${ }^{1}$ Steven J. Jacobs, ${ }^{1}$ Julie S. Moyers, ${ }^{1}$ Rebecca A. Owens, ${ }^{1}$ Aaron D. Showalter, ${ }^{1}$ Martin B. Brenner, ${ }^{2}$ Achim Raap, ${ }^{2}$ Jesper Gromada, ${ }^{2}$ Brian R. Berridge, ${ }^{3}$ David K. B. Monteith, ${ }^{3}$ Niels Porksen, ${ }^{4}$ Robert A. McKay, ${ }^{5}$ Brett P. Monia, ${ }^{5}$ Sanjay Bhanot, ${ }^{5}$ Lynnetta M. Watts, ${ }^{5}$ and M. Dodson Michael ${ }^{1}$

${ }^{1}$ Endocrine Discovery, Eli Lilly and Company, Indianapolis, Indiana, USA. ${ }^{2}$ Endocrine Discovery, Eli Lilly and Company, Hamburg, Germany. ${ }^{3}$ Pathology and Toxicology, Eli Lilly and Company, Greenfield, Indiana, USA. ${ }^{4}$ Clinical Pharmacology, Eli Lilly and Company, Indianapolis, Indiana, USA. ${ }^{5}$ sis Pharmaceuticals, Carlsbad, California, USA.

\begin{abstract}
Uncontrolled hepatic glucose production contributes significantly to hyperglycemia in patients with type 2 diabetes. Hyperglucagonemia is implicated in the etiology of this condition; however, effective therapies to block glucagon signaling and thereby regulate glucose metabolism do not exist. To determine the extent to which blocking glucagon action would reverse hyperglycemia, we targeted the glucagon receptor (GCGR) in rodent models of type 2 diabetes using 2 '-methoxyethyl-modified phosphorothioate-antisense oligonucleotide (ASO) inhibitors. Treatment with GCGR ASOs decreased GCGR expression, normalized blood glucose, improved glucose tolerance, and preserved insulin secretion. Importantly, in addition to decreasing expression of cAMP-regulated genes in liver and preventing glucagon-mediated hepatic glucose production, GCGR inhibition increased serum concentrations of active glucagon-like peptide-1 (GLP-1) and insulin levels in pancreatic islets. Together, these studies identify a novel mechanism whereby GCGR inhibitors reverse the diabetes phenotype by the dual action of decreasing hepatic glucose production and improving pancreatic $\beta$ cell function.
\end{abstract}

\section{Introduction}

Under normal physiologic conditions, glucagon secretion is regulated by changes in systemic glucose and insulin concentrations. Unger hypothesized that disruption of the glucagon-insulin bihormonal relationship contributes to the manifestation of diabetes mellitus (1). Several studies have since tested this hypothesis, and accumulating evidence supports a pathophysiological role of glucagon in the development and progression of diabetes. Basal glucagon is inappropriately elevated (2), and its suppression is impaired following food consumption (3) in type 2 diabetes. Increased hepatic glucose production resulting from elevated glucagon and/or dysregulation of postprandial glucagon secretion likely contribute to hyperglycemia and worsen glucose tolerance in type 2 diabetes.

Pharmacological intervention to suppress glucagon activity is proposed to improve insulin action in the liver and help restore normal hepatic glucose metabolism, thus decreasing hypergly-

Nonstandard abbreviations used: antisense oligonucleotide (ASO); dipeptidyl peptidase (DPP); glucagon-like peptide-1 (GLP-1); glucagon receptor (GCGR); glycerol kinase (GK); glycogen phosphorylase (GP); prohormone convertase-2 (PC2), reverse transcription (RT); Sprague Dawley (SD), streptozotocin (STZ); Zucker diabetic fatty (ZDF)

Conflict of interest: Brian R. Berridge, Diane M. Bodenmiller, Martin B. Brenner, Julia Xiao-Chun Cao, Amy L. Cox, Jesper Gromada, Steven J. Jacobs, M. Dodson Michael, David K. B. Monteith, Julie S. Moyers, Rebecca A. Owens, Niels Porksen, Achim Raap, Aaron D. Showalter, Angela M. Siesky, Kyle W. Sloop, and Hong Yan Zhang are currently employees of Eli Lilly and Co. and may own Eli Lilly and Co. stock and/or stock options. Sanjay Bhanot, Robert A. McKay, Brett P. Monia, and Lynnetta M. Watts are currently employees of Isis Pharmaceuticals and may own Isis Pharmaceuticals stock and/or stock options.

Citation for this article: J. Clin. Invest. 113:1571-1581 (2004). doi:10.1172/JCI200420911. cemia. Glucagon analogs that act as competitive antagonists, including [ $N \alpha$-trinitrophenyl-His1, homo-Arg12]glucagon, [desHis1,Glu9] glucagon-NH2, and [des-His1, des-Phe6,Glu9]glucagon$\mathrm{NH} 2$, transiently lower blood glucose in streptozotocin-induced (STZ-induced) diabetic rats (4-6). Antiglucagon mAbs improve glycemia in STZ-induced diabetic rats, alloxan-induced diabetic rabbits, and ob/ob mice (7-9). Further, Bayer 27-9955, a small molecular weight competitive glucagon receptor (GCGR) antagonist, is efficacious in glucagon challenge experiments in healthy adult humans (10). However, efficacy data from chronic studies in diabetic rodents or humans administered this molecule have not been disclosed.

Recently, mice have been generated lacking GCGR $(11,12)$ or the enzyme required to process functional glucagon, prohormone convertase 2 (PC2) (13). Glucose homeostasis is relatively normal in these animals, yet both plasma glucose and insulin are slightly reduced. In addition, deletion of either gene results in $\alpha$-cell hyperplasia, with Gcgr KO mice also displaying hyperglucagonemia (11-13). These data provide information about the physiological effects resulting from inhibition of glucagon signaling but do not indicate whether inhibiting this pathway will improve glucose control in diabetes.

To test the hypothesis that inhibiting glucagon action will decrease hepatic glucose output and reduce hyperglycemia in type 2 diabetes, we identified and tested 2'-methoxyethyl modified phosphorothioate GCGR antisense oligonucleotides (ASOs). Exploiting modified ASOs of this class to inhibit the GCGR is advantageous over other chemical platforms because these compounds effectively decrease expression of targeted genes in specific tissues such as the liver (14). In addition, these molecules possess extended half-lives that minimize compound dosing regimens (15). The inherent specificity and 
in vivo stability of antisense inhibitors enables characterization of the biological effects of inhibiting the GCGR in type 2 diabetes.

\section{Results}

GCGR ASOs lower plasma glucose in diabetic rodents. Several potent GCGR ASO inhibitors were identified and characterized in doseresponse studies using primary mouse and rat hepatocytes (Figure 1, $A$ and B). To test the efficacy of GCGR ASOs to treat hyperglycemia, $7-8$ week-old $o b / o b$ and $d b / d b$ mice were dosed two times per week with either GCGR ASOs $(148359,180475)$, a generic control ASO (141923) whose sequence does not match any known transcripts in the mouse or rat genomes, a mismatch GCGR ASO (298682) whose sequence is identical to GCGR ASO 180475 except for 7 internal bases, or saline for 4 weeks (Figure 1C, Table 1, and data not shown). Whereas hyperglycemia continued to worsen over time in saline- and control ASO-treated $o b / o b$ mice, animals treated with GCGR ASOs showed a dramatic reduction in plasma glucose. In fact, GCGR ASO 180475 lowered glucose into the normal range. At the end of the 4week treatment period, liver GCGR mRNA was reduced by $85-95 \%$ (Figure 1D). Similar glucose lowering efficacy and target reduction were observed in $d b / d b$ mice undergoing GCGR ASO treatment compared with administration of saline, the generic control ASO, or the mismatch GCGR ASO (Table 1 and data not shown); furthermore, plasma triglycerides in $d b / d b$ mice were lowered following GCGR ASO treatment (Table 1). Interestingly, the lowering of plasma triglyceride levels was paralleled by similar decreases in liver triglyceride content in some animals administered GCGR ASOs (Table 1 and data not shown). The reduction in plasma glucose and triglycerides in $d b / d b$ mice by GCGR ASO 180475 is similar to that reported in studies testing GCGR ASO 148359 for 3 weeks (16). A comparison of metabolic parameters from normal and diabetic rodents treated for 4 weeks with GCGR ASOs is presented in Table 1.

The time-dependent antidiabetic properties of GCGR ASOs were further investigated in a more detailed time course analysis of glucose lowering and target reduction in 8-week-old Zucker diabetic fatty (ZDF) rats. Animals were dosed for 4 weeks with either the control ASO or GCGR ASO 180475 followed by a 4-week washout period during which there was no treatment. Liver GCGR mRNA decreased dramatically to $50 \%$ of that in controls within 24 hours after the first dose of GCGR ASO and to $70 \%$ of controls 48 hours after the sev-
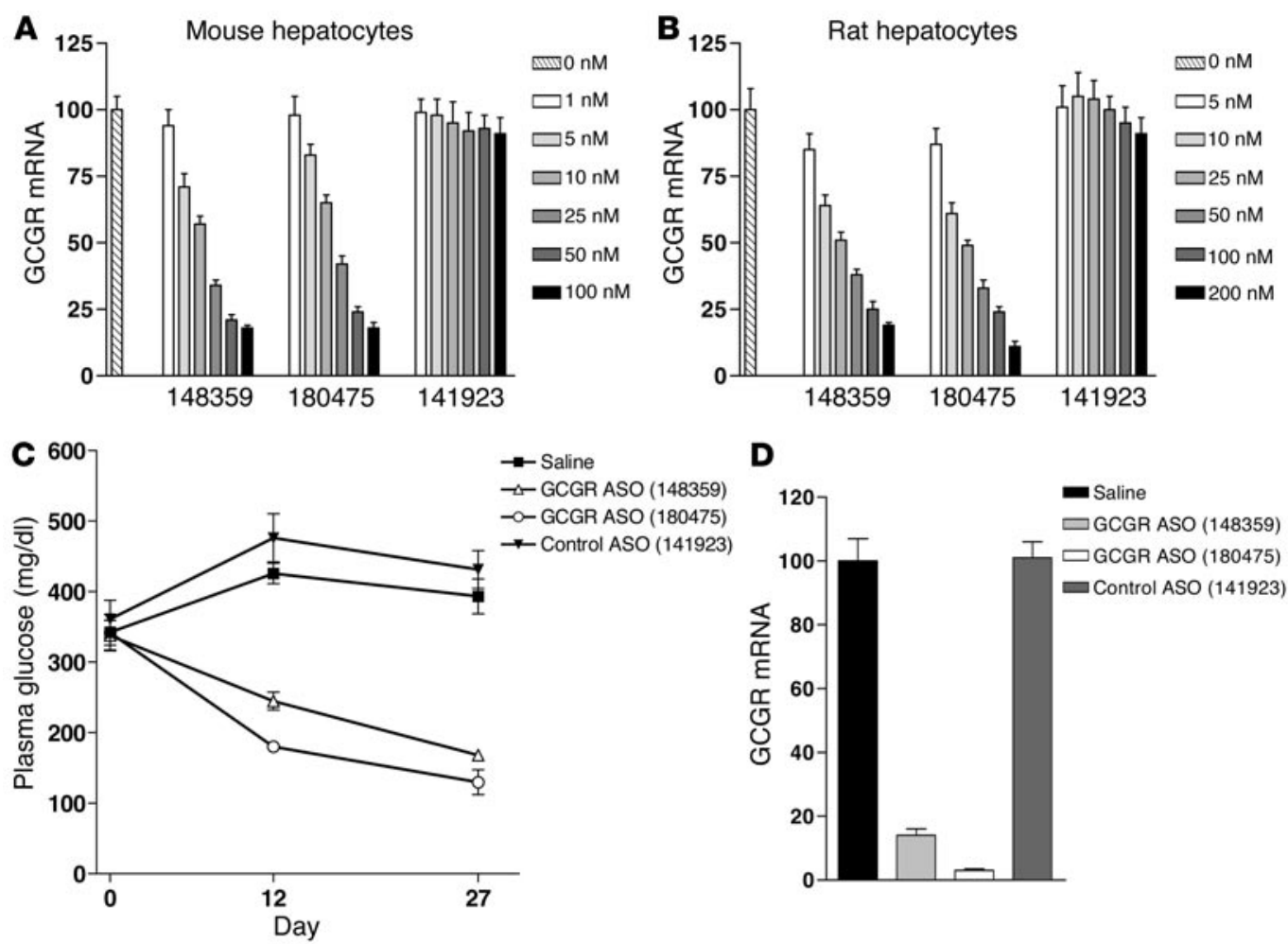

\section{Figure 1}

GCGR ASOs decrease GCGR mRNA and lower plasma glucose in ob/ob mice. Mouse (A) or rat (B) primary hepatocytes were treated with the indicated ASO concentration for 4 hours as described in Methods. Following oligonucleotide treatment, cells were cultured for an additional 16-18 hours. Total RNA was extracted, and GCGR mRNA expression was assessed by RT-PCR. GCGR expression was normalized to total RNA in the same samples using Ribogreen. Data are expressed as percent of saline-treated controls. (C) Nonfasted plasma glucose in 8-week-old male ob/ob mice treated twice per week (every 3.5 days) by subcutaneous injection with saline (filled squares), GCGR ASO 148359 (open triangles), GCGR ASO 180475 (open circles), or control ASO 141923 (filled inverted triangles) for 4 weeks. All ASOs were administered at 25 mg/kg. Data are the mean values \pm SEM of eight mice per treatment group. In overall comparisons, glucose lowering in animals treated with GCGR ASO 148359 and GCGR ASO 180475 was significantly different compared individually with saline- and control ASO-treated animals $(P<0.05$ adjusted using Tukey's $t$ test). (D) Liver GCGR mRNA reduction in ob/ob mice. GCGR mRNA was measured by real-time quantitative RT-PCR from livers of ob/ob mice treated for 4 weeks [i.e., end of the treatment period described in (C)] with saline (black bar), GCGR ASO 148359 (light gray bar), GCGR ASO 180475 (white bar), and control ASO 141923 (dark gray bar). GCGR mRNA was normalized to total RNA in the same samples using Ribogreen. Data are the mean values \pm SEM of four mice per treatment group $(P<0.05$ using Student's $t$ test). 
Table 1

Metabolic parameters of GCGR ASO-treated rodents

\begin{tabular}{|c|c|c|c|c|c|c|c|}
\hline & $\begin{array}{l}\text { Body weight } \\
\text { (g) }\end{array}$ & $\begin{array}{l}\text { Plasma glucose } \\
\text { (mg/dl) }\end{array}$ & $\begin{array}{c}\text { Plasma triglycerides } \\
\text { (mg/dl) }\end{array}$ & $\begin{array}{l}\text { Liver triglycerides } \\
\qquad(\mathrm{mg} / \mathrm{g})\end{array}$ & $\begin{array}{l}\text { Plasma insulin } \\
\text { (ng/ml) }\end{array}$ & $\begin{array}{c}\text { Plasma glucagon } \\
(\mathrm{pg} / \mathrm{ml})\end{array}$ & $\begin{array}{c}\text { Plasma active } \\
\text { GLP-1 (pM) }\end{array}$ \\
\hline \multicolumn{8}{|l|}{ ob/ob mice } \\
\hline Saline & $54.3 \pm 1.6$ & $448 \pm 64$ & $148 \pm 28$ & $220 \pm 27$ & $41.2 \pm 9.4$ & $566 \pm 46$ & $19.6 \pm 7.2$ \\
\hline GCGR ASO & $53.0 \pm 1.9$ & $144 \pm 7^{A}$ & $100 \pm 8$ & $87 \pm 7^{A}$ & $33.0 \pm 6.0$ & $10581 \pm 2479$ & $274.7 \pm 46.8^{A}$ \\
\hline \multicolumn{8}{|l|}{$d b / d b$ mice } \\
\hline Saline & $42.9 \pm 1.5$ & $631 \pm 64$ & $361 \pm 58$ & n.d. & $9.7 \pm 2.4$ & $252 \pm 69$ & $7.7 \pm 0.7$ \\
\hline Control ASO & $44.7 \pm 1.1$ & $642 \pm 47$ & $274 \pm 33$ & n.d. & $12.6 \pm 2.3$ & $352 \pm 59$ & $5.5 \pm 1.0$ \\
\hline GCGR ASO & $46.4 \pm 0.5$ & $220 \pm 14^{A}$ & $97 \pm 7^{A}$ & n.d. & $12.2 \pm 2.8$ & $7265 \pm 415^{A}$ & $75.7 \pm 13.9^{A}$ \\
\hline \multicolumn{8}{|l|}{$d b^{+/ ?}$ lean mice } \\
\hline Control ASO & $28.0 \pm 1.0$ & $196 \pm 12$ & $121 \pm 7$ & $9.1 \pm 1.1$ & n.d. & $80 \pm 1$ & n.d. \\
\hline GCGR ASO & $27.6 \pm 1.0$ & $164 \pm 4^{A}$ & $83 \pm 6^{A}$ & $7.1 \pm 0.6$ & n.d. & $362 \pm 40^{A}$ & n.d. \\
\hline \multicolumn{8}{|l|}{ ZDF rats } \\
\hline Control ASO & $403 \pm 12$ & $417 \pm 38$ & $640 \pm 105$ & $23.5 \pm 1.6$ & $5.0 \pm 1.9$ & $136 \pm 7$ & $17.0 \pm 2.7$ \\
\hline GCGR ASO & $404 \pm 8$ & $143 \pm 15^{A}$ & $250 \pm 25^{A}$ & $17.0 \pm 2.3$ & $4.4 \pm 0.5$ & $548 \pm 20^{A}$ & $184.0 \pm 8.4^{A}$ \\
\hline \multicolumn{8}{|l|}{ SD rats } \\
\hline Saline & $344 \pm 4$ & $116 \pm 3$ & $106 \pm 26$ & $10.3 \pm 0.6$ & $2.7 \pm 0.3$ & $56 \pm 11$ & $12.1 \pm 0.2$ \\
\hline GCGR ASO & $327 \pm 5$ & $104 \pm 7$ & $139 \pm 58$ & $12.3 \pm 1.5$ & $1.3 \pm 0.2^{\mathrm{A}}$ & $855 \pm 122^{A}$ & $113 \pm 21^{A}$ \\
\hline
\end{tabular}

All data are from animals that were treated twice per week (every 3.5 days) by subcutaneous injection with saline, GCGR ASO 180475 , or Control ASO 141923 for 9 total doses. ${ }^{A} P<0.05$. n.d., not determined.

enth dose (Figure 2A). This was paralleled by a significant drop in plasma glucose within 48 hours after the initial GCGR ASO dose and reached maximal efficacy 48 hours after the seventh dose (Figure 2B). During the washout phase, hyperglycemia and GCGR expression in the liver began to rebound within 10 days, but even one month after the final dose, efficacy was still observed as plasma glucose and target mRNA levels in washout animals remained below pretreatment levels (Figure 2, A and B). Glucose lowering achieved by the twice per week dosing schedule and the gradual rebound of GCGR mRNA during the washout period are both consistent with the extended half-lives of 2'-methoxyethyl-modified phosphorothioate ASOs, which typically range from 9 to 19 days $(14,15)$. Plasma insulin levels fell during the treatment phase in both control and GCGR ASO-treated animals (Figure 2C). In the control group, falling insulin levels occurred as glucose levels rose. This is likely the result of progressive $\beta$ cell failure, which typically occurs in ZDF rats between 8 and 12 weeks of age (17). In contrast, in GCGR ASO-treated ZDF rats, insulin levels fell as glucose levels also decreased (Figure 2C). Here, this finding is likely a result of reduced glycemic stimulus rather than $\beta$ cell failure. Importantly, the very robust initial insulin response to a mild rise in
A

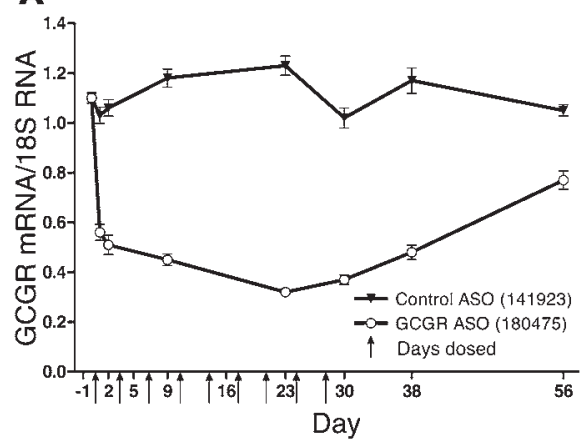

B

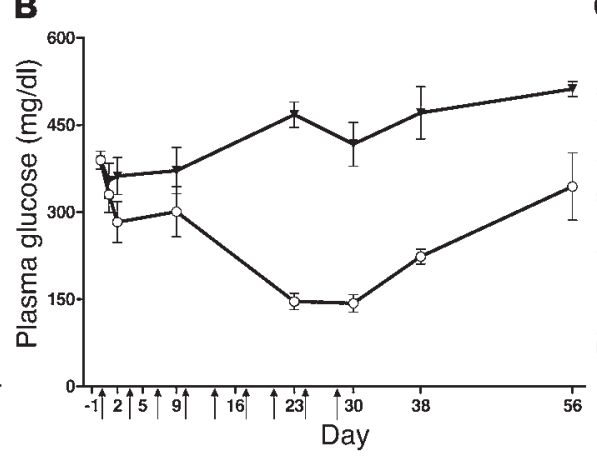

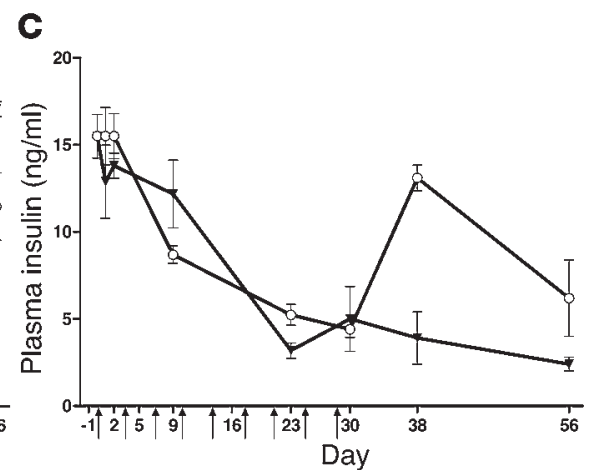

Figure 2

Time course analysis of GCGR mRNA reduction and plasma glucose lowering in ZDF rats. (A) Liver GCGR mRNA reduction in ZDF rats. Seven-week-old male ZDF rats were treated twice per week (every 3.5 days) by subcutaneous injection with control ASO 141923 (filled inverted triangles) or GCGR ASO 180475 (open circles) at $25 \mathrm{mg} / \mathrm{kg}$ for 9 doses (last treatment on day 28) followed by a washout period of equal duration. GCGR mRNA was measured by real-time quantitative RT-PCR from livers of 5 animals removed from the study at each time point. Eukaryotic 18S ribosomal RNA was measured and used to normalize RNA input. Data are the mean values \pm SEM of five rats per treatment group. In overall comparisons during the treatment period, target reduction by GCGR ASO 180475 was significantly different when compared with that in control ASO-treated animals ( $P<0.05$ adjusted using Tukey's $t$ test). (B) Nonfasted plasma glucose in ZDF rats treated as described in (A). Data are the mean values \pm SEM of five rats per treatment group. In overall comparisons during the treatment period, glucose-lowering by GCGR ASO treatment showed significant differences when compared with that in control ASO-treated animals $(P<0.05$ adjusted using Tukey's $t$ test). (C) Nonfasted plasma insulin in ZDF rats treated as described in (A). Data are the mean values \pm SEM of five rats per treatment group. No significant changes were observed during the treatment period; however, individual comparisons between GCGR ASO- and control ASO-treated animals on days 38 and 56 (washout period) were significant $(P<0.05)$. 

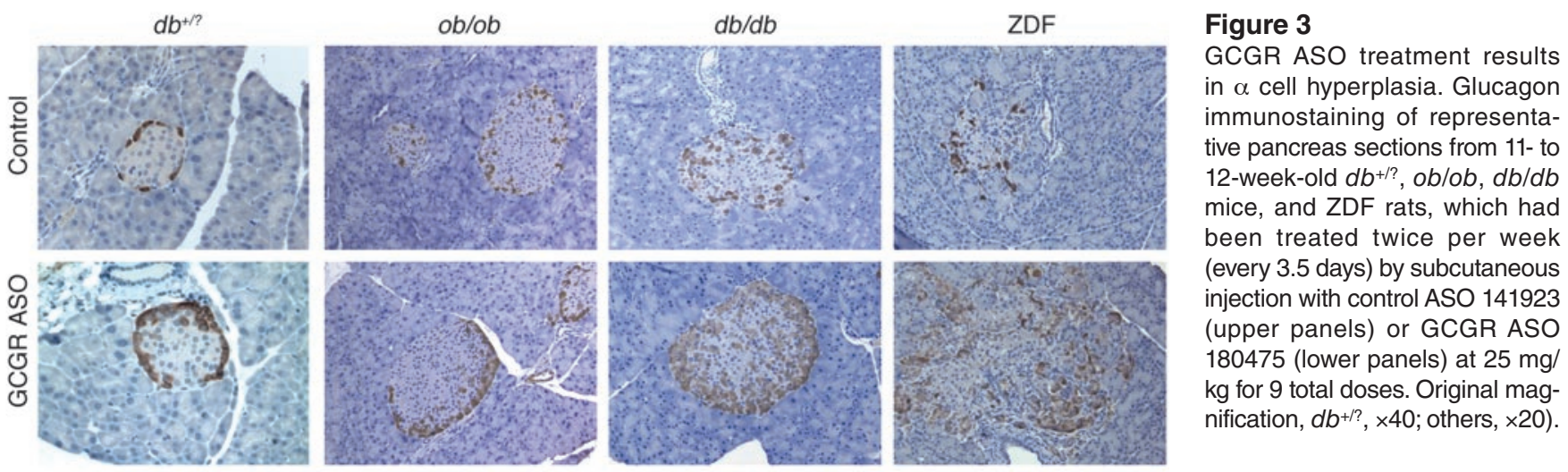

plasma glucose in the GCGR ASO-treated rats during the washout phase is consistent with improved $\beta$ cell function compared with that in the control animals. Further evidence for this is presented below (Figures 8 and 9).

GCGR ASO treatment results in $\alpha$-cell bypertropby, byperplasia, and reversible byperglucagonemia. In addition to dramatic effects on glucose lowering, GCGR ASO treatment resulted in marked hyperglucagonemia in both normal and diabetic rodents (Table 1). This level of hyperglucagonemia is similar to that observed in Gcgr $\mathrm{KO}$ mice $(11,12)$. Elevated concentrations of plasma glucagon in GCGR ASO-treated rodents were accompanied by pancreatic $\alpha$ cell hypertrophy, and $\alpha$ cell hyperplasia was observed in some models (Figure 3 ). $\alpha$-cell hypertrophy, characterized by an increase in individual cell size without an appreciable change in cell number, was present in islets of $d b^{+/}$? lean and $o b / o b$ mice treated for 30 days (Figure 3 ). In addition to displaying $\alpha$ cell hypertrophy, $d b / d b$ mice and ZDF rats exhibited $\alpha$ cell hyperplasia characterized by increased numbers of hypertrophied $\alpha$ cells distributed most prominently along the periphery of individual islets but also extending into central regions of islets (Figure 3). Further characterization was performed by counting glucagon-stained cells of islets from saline or GCGR ASO-treated $d b / d b$ mice. The first 10 islets encountered in individual sections from control and treated animals ( $n=5$ animals per group) were selected for analysis regardless of appearance, and images were captured digitally. Glucagon immunopositive and immunonegative islet cells were manually counted. The islets from GCGR ASO-treated $d b / d b$ mice showed an approximately 2 -fold increase in the number of glucagon positive cells (saline, $28.1 \pm 2.5 \%$ of islet cells; GCGR ASO, $53.5 \pm 1.7 \%$ of islet cells). In addition, pancreatic islets in animals with $\alpha$ cell hyperplasia were qualitatively enlarged and, in the case of ZDF rats, more irregular in contour compared with their saline-treated counterparts. Although GCGR mRNA was reduced in islets isolated from GCGRASO-treated $d b / d b$ mice (Figure $8 \mathrm{C}$ ), there is little evidence supporting direct ASO-mediated pharmacology in these cells (our unpublished data). Because $\alpha$ cells are believed to have fewer GCGRs compared with other islet cell types (18), the increased $\alpha$ cell population in treated animals could account for the measured differences. Alternatively, lower levels of islet GCGRs may have been the result of a compensatory response to hyperglucagonemia.

Treatment of $d b^{+/}$? lean mice for 13 weeks with GCGR ASOs resulted in a remarkable 30 -fold induction of plasma glucagon that returned to normal following an 8-week washout period (Figure 4B), at which time GCGR mRNA levels in the liver also normalized (Figure 4C). During the treatment period, hyperglucagonemia
A

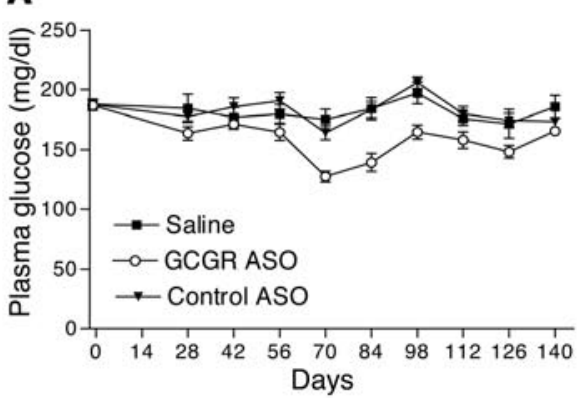

B

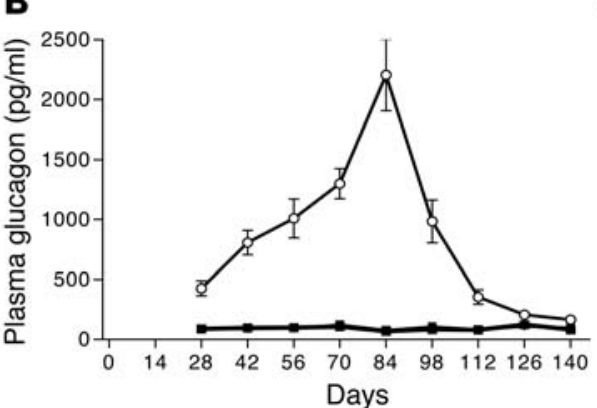

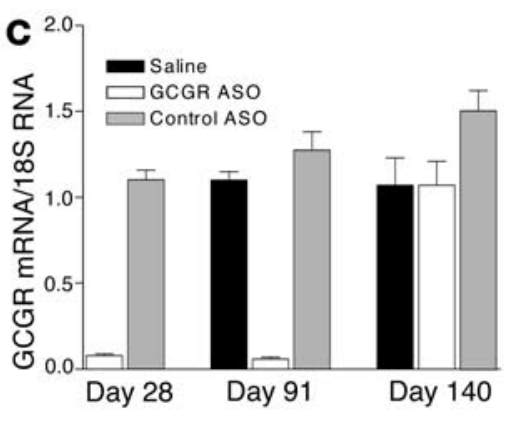

Figure 4

Hyperglucagonemia induced by GCGR ASO treatment is reversible. (A) Seven-week-old male $d b^{+/ ?}$ mice were treated twice per week (every 3.5 days) by subcutaneous injection with saline (filled squares), GCGR ASO 180475 (open circles), or control ASO 141923 (filled inverted triangles). ASOs were administered at $25 \mathrm{mg} / \mathrm{kg}$ for the first 9 doses and at $10 \mathrm{mg} / \mathrm{kg}$ for an additional 18 doses (last treatment on day 91) followed by a treatment-free washout period of 8 weeks. Nonfasted plasma glucose was measured every two weeks, and the data are expressed as the mean \pm SEM of 8 mice per treatment group. (B) Nonfasted plasma glucagon was measured every two weeks from the animals described in (A), and the data are expressed as the mean \pm SEM of 8 mice per treatment group. (C) GCGR mRNA was measured by real-time quantitative RT-PCR from the livers of 5 animals removed from the study at the time points indicated. Eukaryotic $18 \mathrm{~S}$ ribosomal RNA was measured and used to normalize RNA input. Data are the mean values \pm SEM of 5 mice per treatment group $(P<0.05$ using the Student's $t$ test). 


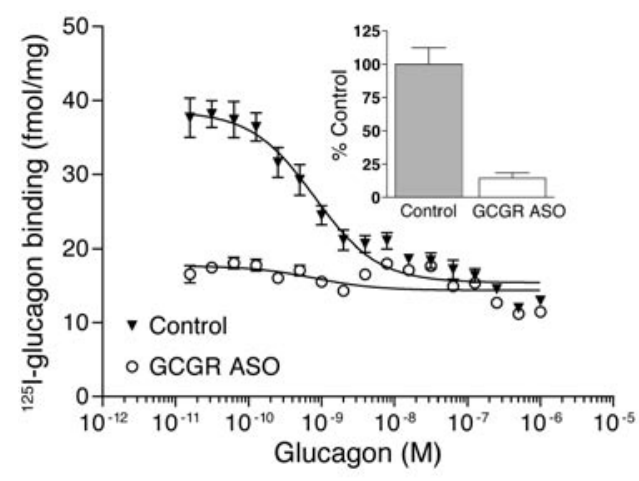

\section{Figure 5}

GCGR ASO treatment decreases glucagon binding to liver membranes. Glucagon binding to liver membranes was assessed by homologous competition in the presence of 0.1 nM 125 -labeled glucagon in filter binding analysis as described in Methods. Results are expressed in $\mathrm{fmol} / \mathrm{mg}$ membrane. Each value represents the mean \pm SEM of duplicate conditions from the liver membranes of 11-week-old $d b / d b$ mice $(n=3$ per treatment group), which had been treated twice per week (every 3.5 days) by subcutaneous injection with control ASO 141923 (filled inverted triangles) or GCGR ASO 180475 (open circles). Receptor expression as percentage of control is shown in the inset. The white bar represents the average $B_{\max } \pm$ SEM for GCGR ASO-treated samples versus control ASO (gray bar) $(P<0.05)$. was coincident with pancreatic- $\alpha$ cell hypertrophy and hyperplasia and an equivocal increase in BrdU incorporation in islets of individual $d b^{+/ \text {? }}$ animals at day 91 (data not shown). Importantly, however, there was no increase in the severity of $\alpha$ cell hyperplasia following the 8-week recovery period as BrdU immunolabeling in GCGR ASO-treated animals was indistinguishable from that of saline and control ASO-treated animals (data not shown).

Because very high levels of serum glucagon are present in animals receiving GCGR ASOs, it is possible that these concentrations might induce hyperglycemia, particularly as hepatic GCGR levels gradually return to normal following treatment withdrawal. It is therefore significant that at no time during the treatment or washout periods did animals with hyperglucagonemia exhibit hyperglycemia (Figure 4A). In fact, GCGR ASO-treated animals showed a moderate decrease in fed plasma glucose at all time points tested. This reversal of hyperglucagonemia is analogous to the reversal of $\alpha$ cell hyperplasia observed in Pc2 KO mice treated with exogenous glucagon by mini-osmotic pump (13). Indeed, it appears both glucagon and its receptor must be functional in order to maintain a feedback loop that restrains $\alpha$ cell growth and subsequent glucagon secretion. The exact nature of this feedback loop is unclear, but it is not believed to be driven by hypoglycemia (19).

GCGR ASOs decrease glucagon-stimulated hepatic glucose production without causing bypoglycemia. To assess whether the reduction in GCGR mRNA correlates with a reduction in functional GCGR number, a homologous competition assay was performed using hepatocyte membranes prepared from $d b / d b$ mice treated with control or GCGR ASOs. ${ }^{125}$ I-glucagon binding was effectively competed by increasing concentrations of unlabeled glucagon in control membrane samples (Figure 5). The calculated apparent $\mathrm{B}_{\max }$ was $180.3 \pm 38.7$ fmol receptor $/ \mathrm{mg}$ protein. Binding of glucagon to membrane samples from mice treated with GCGR ASOs was low by comparison, nearing the limits of detection of the assay. The apparent $\mathrm{B}_{\max }$ for these samples was $25.8 \pm 12.9 \mathrm{fmol}$ receptor/mg protein. Thus, functional GCGR expression is decreased approximately $85 \%$ by GCGR ASO therapy and is in accord with quantitative RT-PCR results.

Consistent with a reduction in the number of GCGRs present on the cell surface of hepatocytes, expression levels of key downstream regulatory genes that control glucagon-mediated hepatic glucose production were decreased in Sprague-Dawley (SD) and ZDF rats undergoing GCGR ASO therapy. The mRNA levels of several gluconeogenic and glycogenolytic enzymes, including glucose-6phosphatase, phosphoenolpyruvate carboxykinase, fructose-1,6bisphosphatase, and glycogen phosphorylase were decreased (Figure 6, A and B). Further, transcript levels for these enzymes correlate with levels of liver glycogen. In the fed state, there was no difference in liver glycogen between control and GCGR ASO-treated groups. However, in the fasted state, liver glycogen in GCGR ASO-treated $d b / d b$ mice was maintained at the level of fed animals, whereas in controls, liver glycogen was decreased (Figure 6C). To confirm that GCGR ASO therapy results in decreased glucagon-stimulated glucose output, glucose production was measured from liver slices obtained from SD rats treated with GCGR ASOs. There were no significant difference in basal rates of glucose production between the saline- and GCGR ASO-treated groups (saline $=664.7 \pm 56.8 \mathrm{mg}$ glucose $/ g$ liver $/ 2 \mathrm{~h}$; GCGR ASO = $541.5 \pm 44.3 \mathrm{mg}$ glucose $/ \mathrm{g}$ liver $/ 2$ h). Glucagon stimulated a 3.4-fold increase in glucose production in the saline-treated group $(2267 \pm 350 \mathrm{mg}$ glucose $/ \mathrm{g}$ liver $/ 2 \mathrm{~h}$; $P<0.05)$, whereas it failed to induce a significant change in the ASOtreated animals $(870.4 \pm 111.3 \mathrm{mg}$ glucose $/ \mathrm{g}$ liver $/ 2 \mathrm{~h})$. Glucose production was stimulated 1.9-fold by epinephrine over basal in both groups (saline $=1241 \pm 78 \mathrm{mg}$ glucose $/ \mathrm{g}$ liver $/ 2 \mathrm{~h}$; GCGR ASO = $1058 \pm 87 \mathrm{mg}$ glucose/g liver/2 h). This is in contrast to Gcgr/- mice, which are more sensitive to epinephrine stimulation compared with their WT littermates (12). It is likely that incomplete receptor KO in GCGR ASO-treated animals or embryonic effects in Gcgr null mice account for this difference.

Since glucagon is believed to be essential for maintaining fasting and postprandial glucose homeostasis, GCGR ASOs have the potential to induce hypoglycemia. As mentioned previously, $\mathrm{db}^{+/ \text {? }}$ lean mice treated for 13 weeks with GCGR ASOs had a moderate decrease in fed plasma glucose (Figure 4A). After 4 weeks of GCGR ASO treatment - when GCGR reduction reaches maximal levels $-d b^{+/}$? lean mice were subjected to periods of fasting up to 24 hours (Figure 7). Although the GCGR ASO-treated group displayed a $10-30 \%$ reduction in plasma glucose, at no time did animals reach adverse levels of hypoglycemia. It is likely that GCGR ASO-treated animals avoid hypoglycemia during fasting by maintaining normal catecholamine- and glucocorticoid-stimulated hepatic glucose production, in addition to possessing low numbers of residual GCGRs. This is in contrast to Gcgr KO mice, which become hypoglycemic during periods of fasting (12).

GCGR ASOs improve pancreatic $\beta$ cell function. The intestinally-derived incretin hormone, glucagon-like peptide-1 (GLP-1), has positive effects on glucose-stimulated insulin secretion as well as on $\beta$ cell survival and islet neogenesis $(20,21)$. Gcgr KO mice are reported to have elevated levels of GLP-1 (13). The studies presented here determine that plasma concentrations of active GLP-1 [GLP-1 (7-36 amide) and GLP-1 (7-37)] are increased 10- to 20-fold in both normal and diabetic rodents treated with GCGR ASOs (Table 1). To determine the source of the elevated GLP-1, mRNA was isolated from pancreatic islets and intestines (colon, ileum, and jejunum) from animals treated with 
A

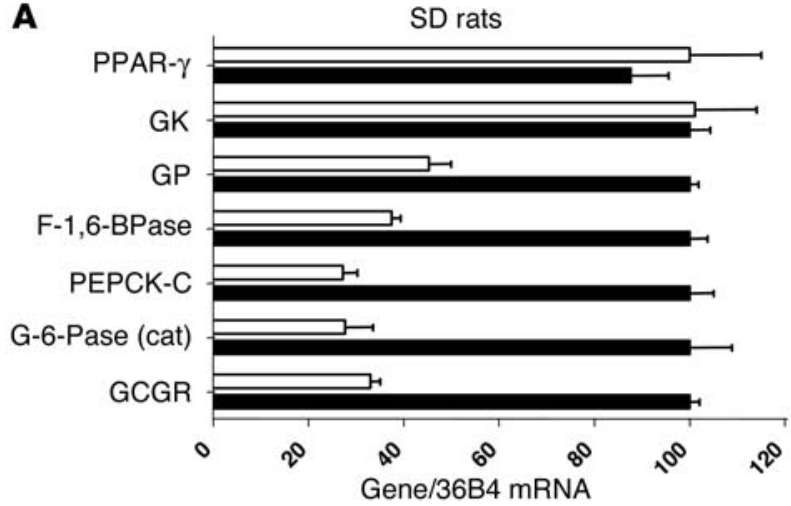

B

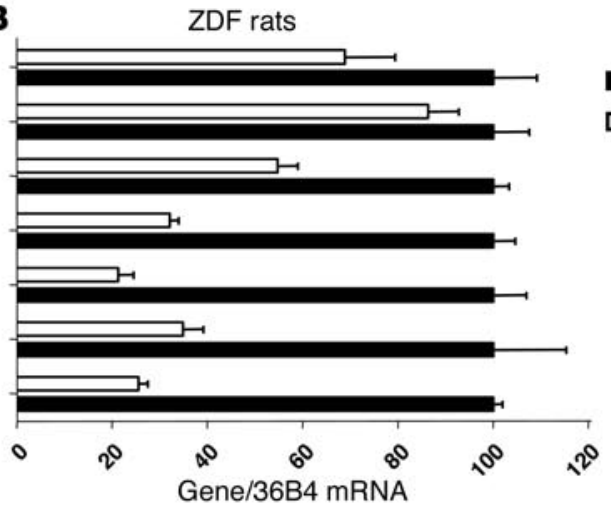

Salline GCGR ASO

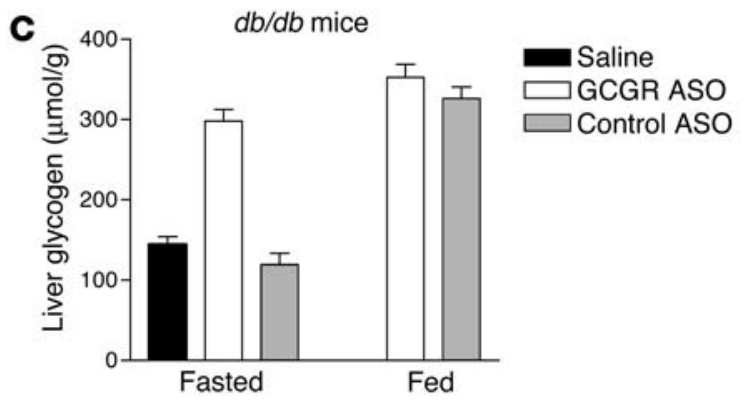

\section{Figure 6}

GCGR ASO treatment affects gluconeogenic and glycogenolytic enzyme gene expression and glycogen content in liver. (A) Expression of genes encoding gluconeogenic and glycogenolytic enzymes in GCGR ASO-treated SD rats. Real-time quantitative RT-PCR was used to profile gene expression from the livers of SD rats treated with GCGR ASO 180475 or saline for 4 weeks. Hepatic GCGR, glucose-6-phosphatase catalytic subunit (G-6-Pase [cat]), phosphoenolpyruvate carboxykinase, cytosolic isoform (PEPCK-C), fructose-1,6-bisphosphatase (F-1,6-Bpase), and glycogen phosphorylase (GP) mRNA levels showed significant differences when compared to control ASO-treated animals $(P<0.05$ using the Student's $t$ test). Differences in the mRNA levels of GK and PPAR $\gamma$ were not observed. Rat 36B4 ribosomal phosphoprotein mRNA was measured and used to normalize RNA input. Data are the mean values \pm SEM of 5 rats per treatment group. (B) Expression of genes encoding gluconeogenic and glycogenolytic enzymes in GCGR ASO-treated ZDF rats. Real-time quantitative RT-PCR was used to profile gene expression from livers of ZDF rats treated as described in (A). (C) Glycogen was measured as described in Methods in liver samples from 11-week-old $d b / d b$ mice ( $n=5$ per treatment group), which had been treated twice per week (every 3.5 days) by subcutaneous injection with saline (black bar), GCGR ASO 180475 (white bar), or control ASO 141923 (gray bar) for 9 total doses. ASOs were administered at 25 mg/kg. GCGR ASO-treated mice had increased liver glycogen in the fasted state $(P<0.05)$ with no significant change in the fed state.

GCGR ASOs, and preproglucagon transcripts were measured by realtime quantitative RT-PCR. Preproglucagon mRNA increased 5-fold in islets from treated animals compared with controls (Figure 8C); however, it was not altered in intestinal tissues (data not shown), suggesting that the increase in plasma GLP-1 is derived from pancreatic $\alpha$ cells. Further, islets isolated from GCGR ASO-treated animals had a 3fold increase in active GLP-1 (Figure 8A), and immunohistochemistry with anti-GLP-1 antibodies identified the presence of GLP-1 in glucagon-stained cells (data not shown). Taken together, these data indicate that pancreatic $\alpha$ cells are the source of the elevated plasma GLP-1 observed in animals treated with GCGR ASOs.

The striking increase in active GLP-1 induced by GCGR ASO therapy would be expected to enhance glucose-stimulated insulin secretion in vivo. To test this hypothesis, intraperitoneal glucose challenges were given to normoglycemic SD rats or 15-weekold ZDF rats undergoing treatment with saline or GCGR ASO 180475. In the basal state, the GCGR ASO-treated SD rats showed no differences in glucose or insulin compared to saline controls (Figure 9, A and B). Following a glucose challenge, glucose excursion in GCGR ASO-treated animals was reduced (Figure 9A), and a robust insulin response was observed (Figure 9B). In ZDF rats, GCGR ASO treatment had a striking effect in normalizing fasting glucose and the glucose excursion curve (Figure 9C); furthermore, basal insulin levels were elevated 2.5-fold by GCGR ASO treatment, and there was a much more pronounced insulin response to a glucose challenge (Figure 9D). The finding that 15 -week-old ZDF rats treated with GCGR ASOs could respond to a glucose challenge prompted analysis of islets of treated animals. Indeed, insulin content and preproinsulin $1 \mathrm{mRNA}$ in islets of $d b / d b$ mice treated with GCGR ASOs were elevated 2-fold (Figure 8, B and C). Taken together, these results suggest that the elevation of active GLP-1 in GCGR ASO-treated animals leads to increased glucose sensitivity of pancreatic $\beta$ cells and promotion of $\beta$ cell survival in these models that undergo an age-dependent islet involution.

Changes in adiposity are not observed in rodents treated with GCGR ASOs. $\mathrm{Gcgr}^{\mathrm{T}}$ - mice have reduced adipose tissue mass, which suggests that glucagon action may be involved in the regulation of whole body composition (12). However, the studies performed here did not identify significant changes in adiposity in animals treated with GCGR ASOs. For example, there was no change in whole body adipose tissue mass in $d b^{+/ \text {? }}$ mice undergoing GCGR ASO treatment for 8 weeks compared with controls (saline: lean mass $=79.65 \%$, adipose mass $=14.87$; GCGR ASO: lean mass $=78.99 \%$, adipose mass $=14.95 \%)$ as assessed by quantitative magnetic resonance 


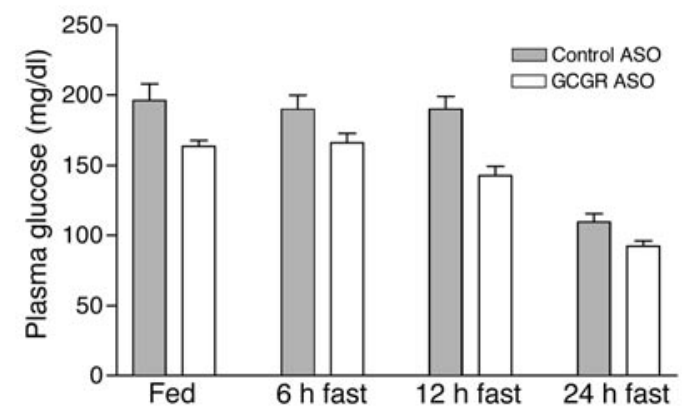

Figure 7

GCGR ASO treatment does not result in adverse hypoglycemia in nondiabetic mice. Nonfasted plasma glucose in 11-week-old male $d b^{+/ \text {? }}$ mice, which had been treated twice per week (every 3.5 days) by subcutaneous injection with control ASO 141923 (gray bars) or GCGR ASO 180475 (white bars) at $25 \mathrm{mg} / \mathrm{kg}$ for a total of 9 doses. Plasma glucose was analyzed in samples obtained from different groups of five animals, which were random fed (6:00 AM sampling) or fasted for 6, 12, or 24 hours (fasting started at 6:00 AM). Data are the mean values \pm SEM of five mice per treatment group. Significant differences $(P<0.05)$ were observed in the fed state and after a 12 hour fast.

analyses (22). Similarly, epididymal white adipose tissue was not reduced in $d b / d b$ mice (saline $=2.27 \pm 0.05 \mathrm{~g}$; GCGR ASO $=2.33 \pm 0.11$ g) treated with GCGR ASOs for 4 weeks. Further, differences in serum levels of circulating free fatty acids in control versus GCGR ASO-treated $d b / d b$ mice (saline $=1.39 \pm 0.05 \mathrm{mEq} / \mathrm{L} ; \mathrm{GCGR}$ ASO $=$ $1.28 \pm 0.09 \mathrm{mEq} / \mathrm{L}$ ) or $o b / o b$ mice (saline $=0.64 \pm 0.1 \mathrm{mEq} / \mathrm{L}$; GCGR $\mathrm{ASO}=0.68 \pm 0.08 \mathrm{mEq} / \mathrm{L}$ ) were not observed. Incomplete receptor $\mathrm{KO}$ in GCGR ASO-treated animals or embryonic effects in $\mathrm{Gcgr}^{-1-}$ mice may account for these differences. Future studies in animal models of diet-induced obesity will be needed to further evaluate the relationship between glucagon action and adiposity.

\section{Discussion}

Because glucagon is elevated in patients with type 2 diabetes (2) and because normalizing glucagon levels during oral glucose challenge tests improves metabolism (3), blocking hepatic glucagon action is likely to improve glycemic control in diabetes. Data reported here support this hypothesis since 2 '-methoxyethyl-modified phosphorothioate-antisense oligonucleotides specific to the GCGR reduce its expression in liver and lower blood glucose in diabetic rodents. Consistent with blocking glucagon action, GCGR ASO therapy decreases glycogenolytic and gluconeogenic enzyme gene expression and prevents glucagon-mediated hepatic glucose production. Importantly, GCGR ASO treatment also leads to an increase in pancreatic $\alpha$ cell expression of active GLP-1. As a result, islet insulin content is increased, insulin secretion is preserved, and glucose tolerance is improved. In addition to identifying mechanisms whereby GCGR ASO inhibitors improve glucose control, the studies presented here demonstrate the long-term effects of targeting glucagon action in several rodent models of type 2 diabetes. Although it is clear that GCGR ASO treatment improves glucose control through hepatic and islet effects, the data do not definitively exclude or support an effect on extrahepatic insulin sensitivity.

Gcgr KO mice have slightly reduced plasma levels of glucose and insulin $(11,12)$; however, the impact of inhibiting this pathway on glucose homeostasis in diabetic models is unclear. Previous studies testing antiglucagon molecules indicate that transient antagonism of glucagon action in the liver would decrease hepatic glucose production $(7,8,10)$. These data provided initial proofof-concept evidence that such molecules might be useful in the treatment of postprandial hyperglycemia and likely have utility in treating impaired glucose tolerance and type 2 diabetes. However, clinical development of small molecular weight and peptide GCGR antagonists has largely failed for technical reasons (23), and thus, studies evaluating the chronic effects of inhibiting this target are needed. As an alternative therapeutic approach, we targeted the GCGR mRNA with specific 2'-methoxyethyl-modified phos-
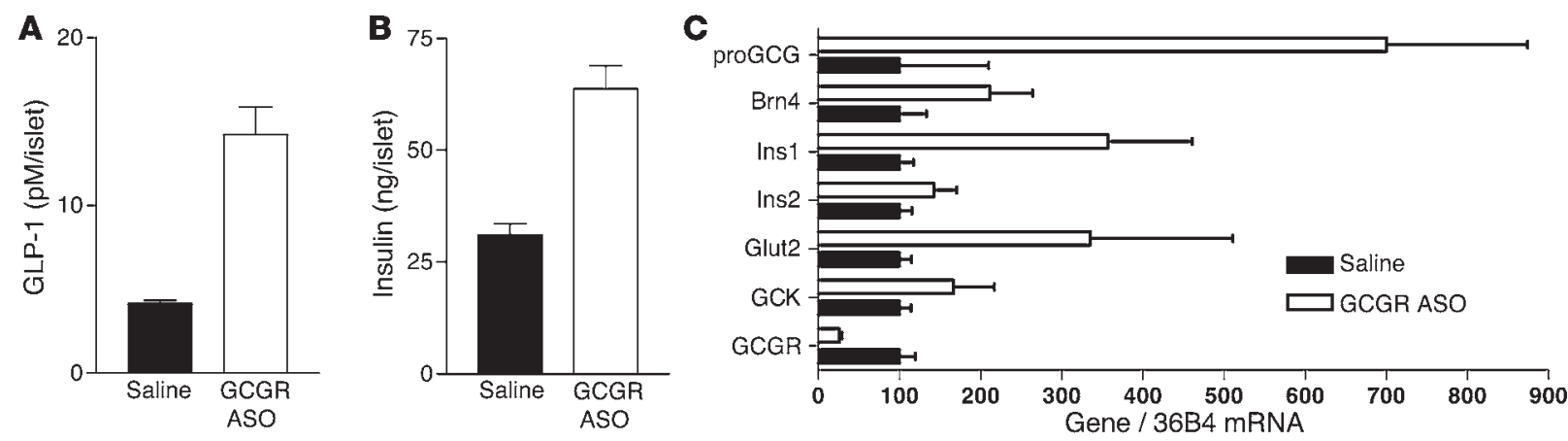

Figure 8

GCGR ASO treatment increases islet GLP-1 and insulin content. (A) Islets were isolated as described in Methods from 12-week-old male $d b / d b$ mice ( $n=5-6$ per treatment group), which had been treated twice per week (every 3.5 days) by subcutaneous injection with saline (black bar) or GCGR ASO 180475 (white bar) at $25 \mathrm{mg} / \mathrm{kg}$ for a total of 9 doses. Five replicates of 10 islets from each animal were extracted with acid ethanol overnight at $4^{\circ} \mathrm{C}$, and GLP-1 was assayed by ELISA. Results are expressed as mean \pm SEM. $P<0.05$. (B) Islet insulin content was assayed by RIA using samples prepared as described in (A). Results are expressed as mean \pm SEM. $P<0.05$. (C) Real-time quantitative RT-PCR was used to profile gene expression from islets of 10-week-old male $d b / d b$ mice $(n=9$ per treatment group), which had been treated twice per week (every 3.5 days) by subcutaneous injection with saline (black bar) or GCGR ASO 180475 (white bar) at $25 \mathrm{mg} / \mathrm{kg}$ for a total of 9 doses. Islets were isolated as described in Methods, and 200 islets from 3 individuals were pooled to give one sample for RNA extraction. Islet GCGR, preproglucagon (proGCG), Brain-4 (Brn4), and insulin-1 (Ins1) levels showed significant differences when compared to saline-treated animals $(P<0.05$ using Student's $t$ test). Differences in the mRNA levels of insulin-2 (Ins2), glucose transporter-2 (Glut2) and glucokinase (GCK) were not observed. Mouse 36B4 ribosomal phosphoprotein mRNA was measured and used to normalize RNA. Data are the mean values \pm SEM of 3 samples per treatment group. 

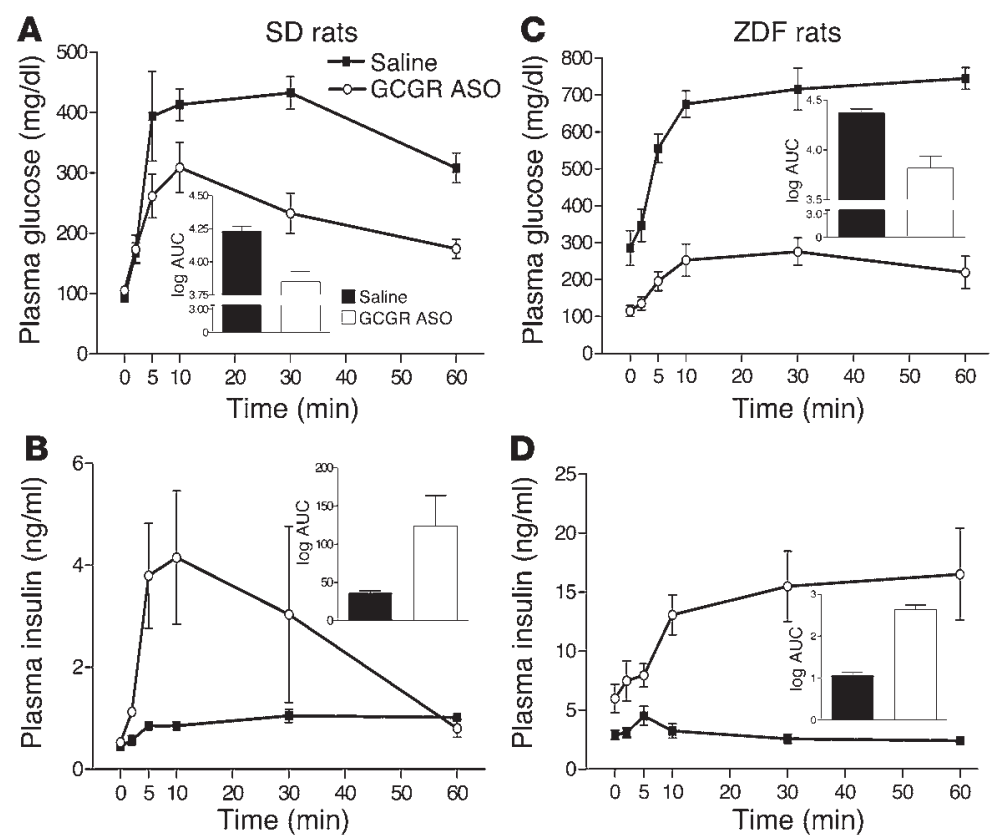

Figure 9

GCGR ASO therapy improves pancreatic $\beta$ cell function. (A) An intraperitoneal glucose challenge ( $2 \mathrm{~g}$ glucose/ $\mathrm{kg}$ body wt) was performed on 9-week-old male SD rats ( $n=5$ per treatment group), which had been treated twice per week (every 3.5 days) by subcutaneous injection with saline (filled squares) or GCGR ASO 180475 (open circles) for 8 doses. ASOs were administered at $25 \mathrm{mg} / \mathrm{kg}$. Blood samples were taken at the indicated time points, and plasma glucose levels were determined. Results are expressed as mean \pm SEM. Inset depicts the log of the area under the glucose excursion curve (AUC) for saline (black bar) and GCGR ASO (white bar). $P<0.05$. (B) Plasma insulin levels for the indicated time points during the glucose challenge described in (A). Results are expressed as mean \pm SEM. Inset depicts the log of the area under the insulin excursion curve for saline (black bar) and GCGR ASO (white bar). $P<0.05$. (C) An intraperitoneal glucose challenge ( $2 \mathrm{~g}$ glucose/kg body wt) was performed on 15 -weekold male ZDF rats ( $n=5$ per treatment group), which had been treated as described in (A). Results are expressed as mean \pm SEM. Inset depicts the log of the area under the glucose excursion curve for saline (black bar) and GCGR ASO (white bar). $P<0.05$. (D) Plasma insulin levels for the indicated time points during the glucose challenge described in (C). Results are expressed as mean \pm SEM. Inset depicts the log of the area under the insulin excursion curve for saline (black bar) and GCGR ASO (white bar). $P<0.05$.

phorothioate ASOs. The use of this class of compound to inhibit the GCGR is advantageous because these molecules effectively decrease gene expression in hepatocytes (14).

Reduced GCGR expression by GCGR ASOs results in $\alpha$ cell hypertrophy, hyperglucagonemia, and, in several cases, $\alpha$ cell hyperplasia, similar to what is observed in GCGR-deficient mice $(11,12)$. Although not observed in either $\mathrm{KO}$ mice or ASO-treated animals, necrolytic migratory erythema - a distinct skin lesion often observed in areas of the lower trunk - is associated with hyperglucagonemia in glucagonoma patients (24). The etiology of this condition is unknown; however, excessive glucagon action in the liver likely contributes to its manifestation because amino acids and other gluconeogenic precursors are mobilized from the periphery to constitutively drive hepatic glucose production. Because glucagon signaling in the liver is blunted by GCGR ASO treatment, the perpetual state of catabolism occurring in glucagonoma patients is unlikely to occur, thus preventing the development of this condition. In addition to elevated glucagon levels, GCGR ASO therapy stimulates pancreatic $\alpha$ cell hyperplasia in some rodent models. Published reports on Gcgr KO mice do not indicate evidence of $\alpha$ cell neoplastic transformation $(11,12)$, and we observed no pancreatic neoplasms in animals treated with GCGR ASOs for 4 months. To date, one report describes loss of function GCGR mutations in humans. Here, a 51-year-old male was found to have hyperglucagonemia and an islet cell pseudoadenoma, both of which were clinically silent $(12,25)$. Accumulating evidence indicates that feedback mechanisms play a role in the plasticity of islet tissues and that hyperplasia may be reversible when feedback inhibition is reestablished. When considering this therapeutic approach for humans, it is important to point out that differences exist across species in the physiological mechanisms that islets utilize to respond to increases in hormone demand. For example, $\beta$ cells within rodent islets predominantly proliferate to meet the insulin need, while in humans, neogenesis (and not proliferation) occurs to satisfy an increasing demand for insulin (26).

Due to the key role that glucagon plays in stimulating hepatic glucose production, other possible adverse effects of targeting the GCGR include hypoglycemia and excessive accumulation of liver glycogen. Importantly, neither condition results from GCGR ASO therapy. Blood glucose levels in treated animals fasted for 24 hours were reduced by only $15 \%$. It is likely that redundancy in counter-regulatory signals such as glucocorticoids and catecholamines compensates for decreased glucagon signaling to maintain glucose levels within the normal range. As expected in the fasted state, hepatic glycogen stores in diabetic rodents undergoing GCGR ASO therapy were higher than those in control animals; however, these levels did not exceed those of fed controls.

In addition to effects in the liver, the studies presented here also demonstrate that inhibiting the GCGR increases circulating levels of the insulinotropic peptide, GLP-1. Normally, biosynthesis of active GLP-1 occurs via specific processing of the proglucagon precursor within enteroendocrine $\mathrm{L}$ cells located in the proximal and distal ends of the small intestine (27). Importantly, however, a small proportion of pancreatic $\alpha$ cell proglucagon is also normally posttranslationally cleaved to yield bioactive GLP-1 $(28,29)$. Analysis of islets and intestinal tissues from GCGR ASOtreated animals revealed that increased pancreatic preproglucagon expression, combined with an expanded $\alpha$ cell population, leads to increased $\alpha$ cell GLP- 1 processing, thereby accounting for the elevated plasma GLP-1 levels. The observance of an increase in GLP-1 in GCGR ASO-treated rodents that possess higher than normal levels of circulating glucagon is similar to findings in STZ-treated rats where elevated glucagon accompanies a concurrent increase in $\alpha$ cell-derived GLP-1 (30).

The ability of GLP-1 to stimulate insulin gene transcription, biosynthesis, and secretion (21), in addition to its involvement in stimulating islet neogenesis and preventing $\beta$ cell apoptosis (20), suggests that therapies tailored to increasing GLP-1 will not only effectively treat the diabetic condition but also may modify or reverse progression of the disease. Indeed, clinical studies testing GLP-1 and GLP-1 mimetics such as AC2993/Exendin-4 and NN2211 have demonstrated effective GLP-1-mediated pharmacology in diabetic 
patients (21). The elevation of GLP-1 in GCGR ASO-treated animals is consistent with the observed pancreatic effects in these animals, including an increase in islet insulin content and the preservation of $\beta$ cell function as demonstrated by an improvement in glucose tolerance with preserved insulin secretion. GLP-1 levels achieved by GCGR ASO therapy are similar to or exceed those shown to be efficacious in humans by exogenous dosing (31) and in rodents via ablation of dipeptidyl peptidase-IV (DPP-IV) activity (32). In addition, because of recent evidence describing intraislet signaling (33), $\alpha$ cell produced GLP- 1 may have local $\beta$ cell effects within islets. This suggests that high levels of circulating GLP-1 may not be needed to achieve efficacy and could ameliorate dose-related side effects associated with exogenous GLP-1 administration.

A reduction in serum and liver triglycerides by GCGR ASO treatment was observed in several studies described here. These results are similar to observations made in $\mathrm{Gggr}^{-1}$ mice (12); however, the mechanisms involved leading to this reduction are unclear at this time. Although this is an exciting result that suggests additional positive effects related to the metabolic syndrome may result from GCGR ASO therapy, studies in additional models will be required to better assess the effects of this treatment on overall lipid metabolism.

Type 2 diabetes mellitus is a progressive disease characterized by impaired insulin secretion, a decline in pancreatic $\beta$ cell function, and chronic insulin resistance in liver and muscle. Because of the central roles that the liver and pancreas play in the control of glucose homeostasis, directly targeting these tissues with therapeutic agents has the potential to modify or delay progression of type 2 diabetes. Molecules such as the GCGR ASO that induce antidiabetic pharmacology at both of these metabolic sites may offer patients more effective treatment options. Clinical studies with human-specific GCGR ASO molecules will be required to test this hypothesis. The successful development of GCGR ASO inhibitors or dual pharmacophores possessing similar combinatorial actions will likely improve glucose control in diabetic patients, while avoiding such complications as hypoglycemia, weight gain, and fluid retention that are often observed with other therapies.

\section{Methods}

Design and identification of lead GCGR ASO inbibitors. A total of 140 ASOs targeting rodent GCGR sequences were designed as 20 base full-phosphorothioate chimeric 2'-O-(2-methoxy)-ethyl modified ASOs. These molecules represent a class of 20 base chimeric ASOs in which an RNase $\mathrm{H}$-sensitive stretch of ten $2^{\prime}$-deoxy residues is flanked on both sides with a stretch of five 2 '-O-(2-methoxy)ethyl modifications. These modifications increase mRNA binding affinity and confer nuclease resistance. Such a chimeric design provides an attractive pharmacological and toxicological profile while maintaining the highly efficient $\mathrm{RNase} \mathrm{H}$ terminating mechanism (34). Following detailed in vitro characterization of the lead molecules from initial screens, rodent GCGR ASO ISIS 148359 (hybridizes to bases 227-246 of mouse GCGR sequence NM 008101.1 and bases 277-296 of rat GCGR sequence M96674.1) and GCGR ASO ISIS 180475 (hybridizes to bases 1348-1367 of mouse GCGR sequence NM 008101.1 and bases $1398-1417$ of rat GCGR sequence M96674.1) were identified as two of the most potent GCGR ASOs. Control ASOs used in these studies were of the same chemistry, design, and length as the active GCGR ASOs. ISIS 141923 is a generic chemistry control that does not have perfect complementarity to any gene in public databases (sequence 5'-CCTTCCCTGAAGGTTCCTCC-3'). ISIS 298682 (sequence 5'-
GCGATTTCCCGTTTTGACCT-3') is a sequence specific 7 base mismatch to GCGR ASO ISIS 180475.

In vitro treatment of primary hepatocytes with GCGR ASOs. GCGR ASOs were screened in primary mouse and rat hepatocytes for their ability to reduce GCGR mRNA levels. Primary hepatocytes were isolated as previously described and plated onto collagen-coated plates (35). Hepatocytes were treated with an ASO and Lipofectin (Invitrogen, Carlsbad, California, USA) mixture for 4 hours in serum-free William's E media (Invitrogen). Following incubation, ASO reaction mixture was replaced with normal growth media (William's E media with 10\% FBS), and cells were incubated under normal conditions for an additional 16-20 hours.

Animal care and treatments. C57Bl/eOlaHsd-Lepob $(o b / o b)$ male mice, $\mathrm{C} 57 \mathrm{Bl} / \mathrm{KsOlaHsd}-L_{e} p_{d b}(d b / d b)$ and lean $\left(d b^{+/}\right)$male mice, and SD male rats were purchased from Harlan (Indianapolis, Indiana, USA). $\mathrm{ZDF} / \mathrm{GmiCrl}-\mathrm{fa} / \mathrm{fa}$ (ZDF) male rats were purchased from Charles River Laboratories (Wilmington, Massachusetts, USA). Animals were acclimated for one week prior to study initiation. Mice were housed five per cage and rats housed one per cage in polycarbonate cages with filter tops. Animals were maintained on a 12:12 hour light-dark cycle (lights on at 6:00 AM) at $21^{\circ} \mathrm{C}$. All animals received deionized water ad libitum. ZDF and SD rats and $d b / d b$ (obese and lean) mice received 5008 Formulab Diet (PMI Nutrition International, Brentwood, Missouri, USA), while $o b / o b$ mice received 5015 Mouse Diet (PMI Nutrition International) ad libitum. All animals were maintained in accordance with the Institutional Animal Use and Care Committee of Eli Lilly and Company and the NIH Guide for the Use and Care of Laboratory Animals. ASOs were prepared in normal saline, and the solution was sterilized through a $0.2 \mu \mathrm{m}$ filter. Animals were dosed with ASO solutions or vehicle (saline) twice per week (separated by 3.5 days) via subcutaneous injection. Before the initiation of each study and once weekly during the study, blood was collected by tail clip without anesthesia into EDTA plasma tubes containing trasylol (Serologicals Proteins, Kankakee, Illinois, USA) and DPP-IV inhibitor (Linco Diagnostic Services, St. Charles, Missouri, USA). Food intake and body weights were measured weekly. For glucose challenge tests, rats were fasted overnight. The next morning, blood samples were taken for determination of fasting glucose and insulin. An intraperitoneal glucose challenge ( $2 \mathrm{~g} / \mathrm{kg}$ body weight) was administered to conscious unrestrained animals, and blood glucose and insulin were measured at 2, 5, 10, 30 , and 60 minutes after glucose injection.

Plasma metabolic measurements. Plasma levels of glucose and triglycerides were determined on the Hitachi 912 clinical chemistry analyzer (Roche Diagnostics, Indianapolis, Indiana, USA). In addition, plasma levels of glucagon, insulin, and active GLP-1 were determined by radioimmunoassay, ELISA, and/or Luminex immunoassay (Linco Diagnostic Services). Cross reactivity of the active GLP-1 ELISA assay with glucagon, GLP-1 1-36 amide, 1-37, 9-36 amide and 9-37, or GLP-2 is undetectable.

Liver glycogen and liver slice methods. For liver glycogen determination, a $100 \mathrm{mg}$ liver sample was digested in $0.5 \mathrm{ml}$ of $1 \mathrm{M} \mathrm{KOH}$ for 30 minutes in a $70^{\circ} \mathrm{C}$ water bath. Samples were vortexed every 10 minutes. Samples were centrifuged briefly to pellet tissue fragments, and a $100 \mu \mathrm{l}$ sample of the supernatant was removed and neutralized with $17 \mu \mathrm{l}$ of $17.4 \mathrm{M}$ acetic acid. Glycogen was enzymatically cleaved to glucose by the addition of $500 \mu \mathrm{l}$ of $0.3 \mathrm{M}$ acetate buffer ( $0.3 \mathrm{M}$ sodium acetate, $0.3 \mathrm{M}$ acetic acid) with $0.5 \%$ amyloglucosidase followed by overnight incubation at $37^{\circ} \mathrm{C}$. Glucose was measured in the supernatant using the Hitachi 912 clini- 
cal chemistry analyzer, and glycogen content was reported as $\mu \mathrm{mol}$ glucose/mg liver tissue. Hepatic glucose production analysis from liver slices was performed immediately following necropsy. Livers were removed and placed in ice-cold slicing buffer [Krebs-Henseleit Bicarbonate Buffer (KHB) pregassed with $\mathrm{O}_{2}, 20 \mathrm{mM}$ glucose, 20 $\mathrm{mM}$ mannitol]. A core was removed from the right lateral lobe using a Tissue Coring Press (Alabama Research and Development, Munford, Alabama, USA), and cores were sliced with a Krumdieck Tissue Slicer (Alabama Research and Development, Munford, Alabama, USA). Slices were transferred, 2 slices per vial, into $2 \mathrm{ml}$ preincubation buffer (KHB, $5.0 \mathrm{mM}$ glucose, $1.0 \mathrm{mM}$ glutamine, $0.5 \mathrm{mM}$ lactate, $34 \mathrm{mM}$ mannitol) and incubated at $29^{\circ} \mathrm{C}$ in a shaking water bath for 1 hour. Slices were transferred 2 slices per vial into $2 \mathrm{ml}$ wash buffer (KHB, $40 \mathrm{mM}$ mannitol) for 20 minutes at $29^{\circ} \mathrm{C}$ in a shaking water bath. Slices were transferred 2 slices per vial into $2 \mathrm{ml}$ of final incubation buffer (KHB, $40 \mathrm{mM}$ mannitol \pm 1 $\mu \mathrm{M}$ glucagon or $5 \mu \mathrm{M}$ epinephrine) and placed back into the $29^{\circ} \mathrm{C}$ shaking water bath. After 2 hours, $100 \mu$ l of final incubation buffer from each vial was analyzed for glucose on the Hitachi 912 clinical chemistry analyzer. Liver slices were weighed, and glucose production per gram of liver tissue was calculated.

Islet isolation and hormone content analyses. Mice were sacrificed by cervical dislocation. The common bile duct was cannulated with a 27-gauge needle- and the pancreas was distended with $3 \mathrm{ml}$ of Hanks' buffer (Sigma-Aldrich, Taufkirchen, Germany), containing 2\% BSA (Applichem, Darmstadt, Germany) and $1 \mathrm{mg} / \mathrm{ml}$ collagenase (Serva, Heidelberg, Germany). Subsequently, the pancreas was removed and digested in Hanks' buffer at $37^{\circ} \mathrm{C}$. Islets were purified on a Histopaque-1077 (Sigma-Aldrich) gradient for 15 minutes at $750 \mathrm{~g}$. Islets were cultured overnight in RPMI-1640 medium containing 10\% FBS, $100 \mathrm{U} / \mathrm{ml}$ penicillin, and $100 \mu \mathrm{g} / \mathrm{ml}$ streptomycin (Invitrogen, Karlsruhe, Germany). For insulin and active GLP-1 content determination, islets were lysed in $75 \%$ ethanol and $1.5 \%$ concentrated $\mathrm{HCl}$. Insulin was determined by radioimmunoassay and GLP-1 by ELISA (Linco Diagnostic Services).

$R N A$ isolation. Total RNA was isolated from primary hepatocytes using the RNeasy 96-kit (QIAGEN Inc., Valencia, California, USA), from isolated islets using TRIzol Reagent (Invitrogen, Carlsbad, California, USA), or from frozen liver tissue by homogenization in Lysing Matrix D shaker tubes (Qbiogene Inc./Bio101 Systems, Carlsbad, California, USA) containing TRIzol Reagent. RNA isolated from tissues was treated with DNase I (Ambion Inc., Austin, Texas, USA), reextracted in TRIzol, and resuspended in DNAse/RNAse-free distilled water (Invitrogen, Carlsbad, California, USA). RNA concentrations were determined from spectrophotometric absorption at $260 \mathrm{~nm}$.

Reverse transcription. Reverse transcription (RT) reactions were performed using the SuperScript First-Strand Synthesis System for RT-PCR (Invitrogen, Carlsbad, California, USA). As per the manufacturer's protocol, $20 \mu \mathrm{l}$ reactions contained RT buffer, 50 units of SuperScript II reverse transcriptase, random hexamers, dNTPs, $\mathrm{MgCl}_{2}$, DTT, RNaseOUT, and 700 ng of total RNA. Two independent RT reactions for each RNA sample were performed. No RT control reactions for each sample contained all reaction components except SuperScript II. Reactions were incubated at $25^{\circ} \mathrm{C}$ for $10 \mathrm{~min}-$ utes, $42^{\circ} \mathrm{C}$ for 50 minutes, and $70^{\circ} \mathrm{C}$ for 15 minutes. Following RT, samples were diluted by adding DNAse/RNAse free distilled water.

Real-time quantitative RT-PCR. Real-time quantitative PCR was performed using the $5^{\prime}$ fluorogenic nuclease assay and an ABI 7900 Prism (PE Applied Biosystems, Foster City, California, USA) to determine the relative abundance of assayed mRNAs. Samples were normalized using Ribogreen (Molecular Probes Inc., Eugene, Oregon, USA) or by determining the relative abundance of either 36B4 mRNA or 18S RNA. The 5' terminus of fluorogenic probes was labeled with FAM (6-carboxy-fluorescein) or VIC and the $3^{\prime}$ terminus contained the quenching dye TAMRA (6-carboxytetramethylrhodamine). Primers and probes were synthesized by Biosearch Technologies Inc. (Novato, California, USA) or PE Applied Biosystems. Primer and probe sequences were as follows: mouse GCGR forward primer 5'-ATTTCCTGCCCCTGGTACCT-3', mouse GCGR reverse primer 5'-CGGGCCCACACCTCTTG -3', mouse GCGR Taqman probe 5'-CCACAAAGTGCAGCACCGCCTAGTGT-3'; mouse 36B4 forward primer 5'-GGCCCGAGAAGACCTCCTT-3', mouse 36B4 reverse primer 5'-TCAATGGTGCCTCTGGAGATT-3', mouse 36B4 Taqman probe 5'-CCAGGCTTTGGGCATCACCACG-3'; rat GCGR forward primer 5'-TCGCGAACCGACCGAT-3', rat GCGR reverse primer 5'-AAAATATCCCTGGCCGATCC-3', rat GCGR Taqman probe 5'-CACCTCTTGGTTCTCGCATCCTCCG-3'; rat 36B4 forward primer 5'-TTCCCACTGGCTGAAAAGGT-3', rat 36B4 reverse primer 5'-GCCGCAGCCGCAAATGC-3', rat 36B4 Taqman probe 5'-AGGCCTTCCTGGCCGATCCATC-3'; rat glycogen phosphorylase (GP) forward primer 5'-TGAACACTATGCGCCTCTGG-3', rat GP reverse primer 5 '-CCGACATTAAAGTCTTGAAGGTTAAA-3', rat GP Taqman probe 5'-CGGCCCGAGCACCCAATGA; rat glycerol kinase (GK) forward primer 5'-GGAGACCAGCCCTGTTAAGCT-3', rat GK reverse primer 5'-GTCCACTGCTCCCACCAATG-3', rat GK Taqman probe 5'-CTGATTTCCATGGCAGCCGCG-3'; rat PPAR $\gamma$ forward primer 5'-GCTGGCCTCCCTGATGAATA-3', rat PPAR $\gamma$ reverse primer 5'-GCTTCCGCAGGCTTTTGA-3', rat PPAR $\gamma$ Taqman probe 5'-AGGGACAAGGATTCATGACCAGGGAGTTC-3'. Taqman primer-probe sets for the following genes were obtained from PE Applied Biosystems (Taqman Assays-on-Demand Gene Expression Products) rat glucose-6-phosphatase catalytic subunit (catalog no. Rn00565347) and 18S rRNA (catalog no. 4310893E). PCR reactions were run in triplicate $20 \mu \mathrm{l}$ reactions that contained Universal Master Mix (PE Applied Biosystems), 4 pmols of each forward and reverse primer, 3 pmols of probe, and cDNA. Two-step PCR cycling was carried out as follows: $50^{\circ} \mathrm{C} 2$ minutes for $1 \mathrm{cycle}, 95^{\circ} \mathrm{C} 10$ minutes for 1 cycle, and $95^{\circ} \mathrm{C} 15$ seconds, $60^{\circ} \mathrm{C} 1$ minute for 40 cycles.

GCGR binding assay. For filtration binding analysis, 15-20 $\mu \mathrm{g}$ of membrane from control ASO- or GCGR ASO-treated $d b / d b$ mice were incubated with $0.1 \mathrm{nM}{ }^{125} \mathrm{I}$-glucagon $(2000 \mathrm{Ci} / \mathrm{mmol}$, PerkinElmer, Boston, Massachusetts, USA) and the indicated concentrations of unlabeled glucagon (Eli Lilly and Co., Indianapolis, Indiana, USA) in buffer containing $50 \mathrm{mM}$ HEPES, $1 \mathrm{mM}$ $\mathrm{MgCl}_{2}, 5$ mM EGTA, 0.005\% Tween 20, 0.1\% BSA, and EDTA-free protease inhibitor cocktail (Roche Diagnostics). Assays were performed under steady state conditions in the presence of excess labeled ligand on 96-well MultiScreen-HV $0.45 \mu \mathrm{m}$ filter plates (Millipore, Bedford, Massachusetts, USA). Following incubation for 2 hours at room temperature, plates were rapidly washed by filtration with ice-cold buffer (20 mM Tris, $\mathrm{pH} 7.4)$ and dried for 45 minutes at $50^{\circ} \mathrm{C}$. Following the addition of Optiphase Supermix (PerkinElmer), plates were counted on a Wallac Microbeta scintillation counter. Data analyses were performed using GraphPad Prism software and expressed as mean \pm SEM. Data obtained for samples from animals treated with GCGR ASOs neared the limits of detection for the assay and curve-fitting parameters. In order to derive a numerical value for apparent $B_{\max }$, the $\mathrm{K}_{\mathrm{d}}$ was fixed at the average value $(0.69 \pm 0.2 \mathrm{nM})$ obtained from the samples from the control ASO-treated animals. 
Immunohistochemistry. Representative sections of the pancreas were fixed in $10 \%$ neutral buffered formalin for 24-36 hours, processed to paraffin embedment, and sectioned at 4 microns. Deparaffinized sections were immunohistochemically labeled for glucagon using an avidin-biotin immunohistochemistry procedure to qualitatively assess pancreatic islet cell populations. Antigen retrieval was accomplished by microwaving in $0.1 \mathrm{M}$ sodium citrate buffer (pH 6.0) (Zymed Laboratories Inc., San Francisco, California, USA) at $98^{\circ} \mathrm{C}$ for 13 minutes. Endogenous peroxidase activity was blocked with $0.3 \%$ hydrogen peroxide for 30 minutes while nonspecific protein binding was blocked with Power Block (Biogenex, San Ramon, California, USA) for 7 minutes. Sections were labeled for glucagon (rabbit polyclonal, 1:200, Dako Corporation, Carpinteria, California, USA) for 30 minutes at room temperature followed by further incubation with a biotinylated secondary antibody for an additional 30 minutes. Signal amplification and visualization were accomplished by incubation in an avidin-biotin complex solution (Vectastain ABC Elite; Vector Laboratories Inc., Burlingame California, USA) and chromogenic development with diaminobenzidine (Vector Laboratories Inc.). Sections were counterstained with Mayer's hematoxylin and permanently mounted.
Statistics. Data are represented as mean \pm SEM and were compared using Student's $t$ test. Repeated measures analysis of variance was used to assess the statistical significance between time courses. Tukey's $t$ test was used to adjust $P$ values for multiple comparisons. The null hypothesis was rejected at $P<0.05$.

\section{Acknowledgments}

We wish to thank José Caro, Jamie Dananberg, Richard DiMarchi, Hans-Juergen Mest, William Roeder, Frank Bennett, and Stan Crooke for their support in pursuing these studies. We also wish to thank Daniel Briere, Joseph Brozinick, Stephen Iturria, Brian Oldham, and Ann Nestorowicz for their advice and technical support.

Received for publication December 22, 2003, and accepted in revised form March 30, 2004.

Address correspondence to: Kyle W. Sloop, Endocrine Discovery, Lilly Research Laboratories, Drop Code 0424, Indianapolis, Indiana 46285, USA. Phone: (317) 651-2856; Fax: (317) 276-9086; E-mail: sloop_kyle_w@lilly.com.
1. Unger, R.H. 1975. Letter: Glucagon in pathogenesis of diabetes. Lancet. 1:1036-1042.

2. Reaven, G.M., Chen, Y.D., Golay, A., Swislocki, A.L., and Jaspan, J.B. 1987. Documentation of hyperglucagonemia throughout the day in nonobese and obese patients with noninsulindependent diabetes mellitus. J. Clin. Endocrinol. Metab. 64:106-110.

3. Shah, P., et al. 2000. Lack of suppression of glucagon contributes to postprandial hyperglycemia in subjects with type 2 diabetes mellitus. J. Clin. Endocrinol. Metab. 85:4053-4059.

4. Johnson, D.G., Goebel, C.U., Hruby, V.J., Bregman, M.D., and Trivedi, D. 1982. Hyperglycemia of diabetic rats decreased by a glucagon receptor antagonist. Science. 215:1115-1116.

5. Unson, C.G., Gurzenda, E.M., and Merrifield, R.B. 1989. Biological activities of desHis1[Glu9]glucagon amide, a glucagon antagonist. Peptides. 10:1171-1177.

6. Van Tine, B.A., et al. 1996. Low level cyclic adenosine $3^{\prime}, 5^{\prime}$-monophosphate accumulation analysis of [des-His1, des- Phe6, Glu9] glucagon$\mathrm{NH} 2$ identifies glucagon antagonists from weak partial agonists/antagonists. Endocrinology. 137:3316-3322.

7. Brand, C.L., et al. 1994. Immunoneutralization of endogenous glucagon with monoclonal glucagon antibody normalizes hyperglycaemia in moderately streptozotocin-diabetic rats. Diabetologia. 37:985-993.

8. Brand, C.L., Jorgensen, P.N., Svendsen, I., and Holst, J.J. 1996. Evidence for a major role for glucagon in regulation of plasma glucose in conscious, nondiabetic, and alloxan-induced diabetic rabbits. Diabetes. 45:1076-1083.

9. Brand, C.L., Hansen, B., Groneman, S., Boysen, M., and Holst, J.J. 2000. Sub-chronic glucagon neutralisation improves diabetes in ob/ob mice [abstract]. Diabetes. 49:A81.

10. Petersen, K.F., and Sullivan, J.T. 2001. Effects of a novel glucagon receptor antagonist (Bay 279955 ) on glucagon-stimulated glucose production in humans. Diabetologia. 44:2018-2024.

11. Parker, J.C., Andrews, K.M., Allen, M.R., Stock, J.L., and McNeish, J.D. 2002. Glycemic control in mice with targeted disruption of the glucagon receptor gene. Biochem. Biophys. Res. Commun.
290:839-843.

12. Gelling, R.W., et al. 2003. Lower blood glucose, hyperglucagonemia, and pancreatic $\alpha$ cell hyperplasia in glucagon receptor knockout mice. Proc. Natl. Acad. Sci. U. S. A. 100:1438-1443.

13. Webb, G.C., Akbar, M.S., Zhao, C., Swift, H.H., and Steiner, D.F. 2002. Glucagon replacement via micro-osmotic pump corrects hypoglycemia and $\alpha$-cell hyperplasia in prohormone convertase 2 knockout mice. Diabetes. 51:398-405.

14. Zhang, H., et al. 2000. Reduction of liver Fas expression by an antisense oligonucleotide protects mice from fulminant hepatitis. Nat. Biotechnol. 18:862-867.

15. Yu, R.Z., et al. 2001. Pharmacokinetics and pharmacodynamics of an antisense phosphorothioate oligonucleotide targeting Fas mRNA in mice. J. Pharmacol. Exp. Ther. 296:388-395.

16. Liang, Y., et al. 2004. Reduction in glucagon receptor expression by an antisense oligonucleotide ameliorates diabetic syndrome in $\mathrm{db} / \mathrm{db}$ mice. Diabetes. 53:410-417.

17. Etgen, G.J., and Oldham, B.A. 2000. Profiling of Zucker diabetic fatty rats in their progression to the overt diabetic state. Metabolism. 49:684-688.

18. Kieffer, T.J., Heller, R.S., Unson, C.G., Weir, G.C., and Habener, J.F. 1996. Distribution of glucagon receptors on hormone-specific endocrine cells of rat pancreatic islets. Endocrinology. 137:5119-5125.

19. Vincent, M., et al. 2003. Abrogation of protein convertase 2 activity results in delayed islet cell differentiation and maturation, increased $\alpha$-cell proliferation, and islet neogenesis. Endocrinology. 144:4061-4069.

20. Drucker, D.J. 2003. Glucagon-like peptides: regulators of cell proliferation, differentiation, and apoptosis. Mol. Endocrinol. 17:161-171.

21. Holz, G.G., and Chepurny, O.G. 2003. Glucagonlike peptide-1 synthetic analogs: new therapeutic agents for use in the treatment of diabetes mellitus. Curr. Med. Chem. 10:2471-2484.

22. Tinsley, F.C., Taicher, G.Z., and Heiman, M.L. 2004. Evaluation of a quantitative magnetic resonance method for mouse whole body composition analysis. Obes. Res. 12:150-160.

23. McCormack, J.G., Westergaard, N., Kristiansen, M., Brand, C.L., and Lau, J. 2001. Pharmacologi- cal approaches to inhibit endogenous glucose production as a means of anti-diabetic therapy. Curr. Pharm. Des. 7:1451-1474.

24. Chastain, M.A. 2001. The glucagonoma syndrome: a review of its features and discussion of new perspectives. Am. J. Med. Sci. 321:306-320.

25. Hansen, L.H., et al. 1999. Identification of a glucagon receptor gene deletion mutation in a patient with hyperglucagonemia and pseudoadenomatous hyperplasia of pancreatic $\alpha$ cells [abstract P3-441]. Programs and Abstracts: 81st Annual Meeting of the Endocrine Society. 441:533.

26. Butler, A.E., et al. 2003. $\beta$-cell deficit and increased $\beta$-cell apoptosis in humans with type 2 diabetes. Diabetes. 52:102-110.

27. Drucker, D.J. 2001. Minireview: the glucagon-like peptides. Endocrinology. 142:521-527.

28. Uttenthal, L.O., et al. 1985. Molecular forms of glucagon-like peptide- 1 in human pancreas and glucagonomas. J Clin. Endocrinol. Metab. 61:472-479.

29. Mojsov, S., Kopczynski, M.G., and Habener, J.F. 1990. Both amidated and nonamidated forms of glucagon-like peptide I are synthesized in the rat intestine and the pancreas. J. Biol. Chem. 265:8001-8008.

30. Nie, Y., et al. 2000. Regulation of pancreatic PC1 and PC2 associated with increased glucagon-like peptide 1 in diabetic rats. J. Clin. Invest. 105:955-965.

31. Ritzel, R., et al. 2001. Glucagon-like peptide 1 increases secretory burst mass of pulsatile insulin secretion in patients with type 2 diabetes and impaired glucose tolerance. Diabetes. 50:776-784.

32. Yasuda, N., Nagakura, T., Yamazaki, K., Inoue, T., and Tanaka, I. 2002. Improvement of high fat-dietinduced insulin resistance in dipeptidyl peptidase IV-deficient Fischer rats. Life Sci. 71:227-238.

33. Cejvan, K., Coy, D.H., and Efendic, S. 2003. Intraislet somatostatin regulates glucagon release via type 2 somatostatin receptors in rats. Diabetes. 52:1176-1181.

34. McKay, R.A., et al. 1999. Characterization of a potent and specific class of antisense oligonucleotide inhibitor of human protein kinase C- $\alpha$ expression. J. Biol. Chem. 274:1715-1722.

35. Crooke, R.M., Graham, M.J., Cooke, M.E., and Crooke, S.T. 1995. In vitro pharmacokinetics of phosphorothioate antisense oligonucleotides. J. Pharmacol. Exp. Ther. 275:462-473. 